\title{
REGION OF STARLIKENESS OF BOUNDED ANALYTIC FUNCTIONS
}

\author{
S. R. KULKARNI AND U. H. NAIK
}

\begin{abstract}
In this paper we obtain the radius of starlikeness for functions of the type $f(z)=a_{1} z+a_{2} z^{2}+\cdots$ which are analytic and univalent in the unit disk and satisfy $0<|f(z)| \leq \alpha$ in $0<|z|<1$, where $\alpha$ is real.
\end{abstract}

\section{Introduction}

Let $\varphi$ denote the class of functions which are analytic and univalent in the unit disk $\mathcal{E}=\{z:|z|<1\}$. A function $f$ in $\varphi$ is said to be starlike of order $\beta$ if and only if $\operatorname{Re}\left[z f^{\prime}(z) / f(z)\right]>\beta \quad(0 \leq \beta<1)$, for $z$ in $\mathcal{E}$. Radius of starlikeness of a function $f$ is the largest $r_{0}, 0<r_{0}<1$ for which it is starlike of order $\beta$ in $|z|<r_{0}$.

\section{Main theorem}

Let $f(z)=a_{1} z+a_{2} z^{2}+\cdots$ be an anlytic and univalent function in $\mathcal{E}$ and $0<$ $|f(z)| \leq \alpha$ in $0<|z|<1$, where $0<a_{1}<\alpha$ and all other coefficients $a_{2}, a_{3}, \ldots$ are complex. Then $f(z)$ is starlike function of order $\beta$, in $|z|<r_{0}$, where $r_{0}$ is the smallest positive root of

$$
r^{3} a_{1} \alpha-r^{2}\left(a_{1}^{2} \beta+2 \alpha^{2}\right)+r(2 \beta+1) a_{1} \alpha+\alpha^{2} \beta=0
$$

Proof. For $\alpha=a_{1}, f(z)=a_{1} z$, the proof is trivial. For general case let us consider

$$
F(z)=\left\{f(z) / \alpha z-a_{1} / \alpha\right\} /\left[1-a_{1} f(z) / \alpha^{2} z\right]
$$

Received February 10, 1993; revised September 26, 1994.

1991 Mathematics Subject Classification. Primary 30C45.

Key words and phrases. Starlike functions, radius of starlikeness, analytic functions, bounded analytic functions. 
Clearly $F(z)$ satisfies Schwarz's condition in $\mathcal{E}$. Hence by Schwarz's lemma

$$
\left|\frac{f(z) / \alpha z-a_{1} / \alpha}{1-a_{1} f(z) / \alpha^{2} z}\right| \leq|z| \quad \text { for all } z \text { in } \mathcal{E}
$$

This on simplification gives us

$$
\begin{aligned}
& |f(z) / z|^{2}-2 a_{1} \operatorname{Re}(f(z) / z)+a_{1}^{2} \\
\leq & \alpha^{2}|z|^{2}\left(1-\left[2 a_{1} / \alpha^{2}\right] \operatorname{Re}(f(z) / z)+\left[a_{1}^{2} / \alpha^{4}\right]|f(z) / z|^{2}\right) .
\end{aligned}
$$

Then we get

$$
\left(1-a_{1}^{2} r^{2} / \alpha^{2}\right)|f(z) / z|^{2}-2\left(1-r^{2}\right) a_{1} \operatorname{Re}(f(z) / z) \leq \alpha^{2} r^{2}-a_{1}^{2},
$$

which is equivalent to

$$
\left|\frac{f(z)}{z}-\frac{\left(1-r^{2}\right) \alpha^{2} a_{1}}{\alpha^{2}-a_{1}^{2} r_{1}^{2}}\right| \leq \frac{\alpha r\left(\alpha^{2}-a_{1}^{2}\right)}{\alpha^{2}-a_{1}^{2} r_{1}^{2}} .
$$

Now let us write

$$
G(z)=f(z) / z
$$

Since $f(z) / \alpha$ satisfies the hypothesis of Schwarz's lemma, we have $|G(z)| \leq \alpha$ and from a well known result

$$
\left|G^{\prime}(z)\right| \leq \alpha\left(1-|G(z)|^{2}\right) /\left(1-r^{2}\right) .
$$

Differentiation of (4) gives us in light of (5)

$$
\left|f^{\prime}(z)-f(z) / z\right| \leq r\left(\alpha-[1 / \alpha]|f(z) / z|^{2}\right) /\left(1-r^{2}\right)
$$

This on simplification gives us

$$
\begin{aligned}
\operatorname{Re}\left\{\frac{z f^{\prime}(z)}{f(z)}\right\} & \geq 1-\frac{r^{2}\left(\alpha-[1 / \alpha]|f(z) / z|^{2}\right)}{|f(z)|\left(1-r^{2}\right)} \\
& =\frac{r}{|f(z)|}\left(|f(z) / z|-\frac{r\left(\alpha-[1 / \alpha]|f(z) / z|^{2}\right)}{\left(1-r^{2}\right)}\right) \\
& \geq \frac{r}{|f(z)|}\left(\operatorname{Re}(f(z) / z)-\frac{r\left(\alpha-[1 / \alpha]|f(z) / z|^{2}\right)}{\left(1-r^{2}\right)}\right) \\
& =\frac{r^{2}}{\alpha|f(z)|\left(1-r^{2}\right)}\left\{\left|\frac{f(z)}{z}\right|^{2}+\frac{\alpha\left(1-r^{2}\right)}{r} \operatorname{Re}\left(\frac{f(z)}{z}\right)-\alpha^{2}\right\} \\
& \geq \frac{r^{2}}{\alpha(\alpha)\left(1-r^{2}\right)}\left\{\left|\frac{f(z)}{z}+\frac{\alpha\left(1-r^{2}\right)}{2 r}\right|^{2}-\frac{\alpha^{2}\left(1+r^{2}\right)^{2}}{4 r^{2}}\right\}
\end{aligned}
$$


Now for $\operatorname{Re}\left\{z f^{\prime}(z) / z\right\}>\beta$, we must have

$$
\left\{\left|\frac{f(z)}{z}+\frac{\alpha\left(1-r^{2}\right)}{2 r}\right|^{2}-\frac{\alpha^{2}\left(1+r^{2}\right)^{2}}{4 r^{2}}\right\}>\frac{\beta \alpha^{2}\left(1-r^{2}\right)}{r^{2}} .
$$

Next we have from (3)

$$
\left|\left\{\frac{f(z)}{z}+\frac{\alpha\left(1-r^{2}\right)}{2 r}\right\}-\left\{\frac{\alpha^{2}\left(1-r^{2}\right) a_{1}}{\alpha^{2}-a_{1}^{2} r^{2}}+\frac{\alpha\left(1-r^{2}\right)}{2 r}\right\}\right| \leq \frac{\alpha r\left(\alpha^{2}-a_{1}^{2}\right)}{\alpha^{2}-a_{1}^{2} r^{2}} .
$$

Which is equivalent to

$$
\begin{aligned}
\left|\frac{f(z)}{z}+\frac{\alpha\left(1-r^{2}\right)}{2 r}\right| & \geq \frac{\alpha^{2}\left(1-r^{2}\right) a_{1}}{\alpha^{2}-a_{1}^{2} r^{2}}+\frac{\alpha\left(1-r^{2}\right)}{2 r}-\frac{\alpha r\left(\alpha^{2}-a_{1}^{2}\right)}{\alpha^{2}-a_{1}^{2} r^{2}} \\
& =\frac{\alpha}{2 r\left(\alpha-a_{1} r\right)}\left(\alpha+a_{1} r-3 \alpha r^{2}+a_{1}^{2} r^{3}\right) .
\end{aligned}
$$

Finally for $\operatorname{Re}\left(z f^{\prime}(z) / z\right)>\beta$, we must have

$$
\frac{\alpha^{2}}{4 r^{2}\left(\alpha-a_{1} r\right)^{2}}\left(\alpha+a_{1} r-3 \alpha r^{2}+a_{1} r^{3}\right) \geq \frac{\beta \alpha^{2}(1-r)^{2}}{r^{2}}+\frac{\alpha^{2}\left(1+r^{2}\right)^{2}}{4 r^{2}},
$$

consequently, we get

$$
\left(\alpha+a_{1} r-3 \alpha r^{2}+a_{1} r^{3}\right)^{2} \geq\left\{4 \beta+1-r^{2}(4 \beta-2)+r^{4}\right\}\left(\alpha-a_{1} r\right)^{2} .
$$

This on simplification gives us

$$
r^{3} a_{1} \alpha-r^{2}\left(a_{1}^{2} \beta+2 \alpha^{2}\right)+r(2 \beta+1) a_{1} \alpha+\alpha^{2} \beta=0 .
$$

Hence the result follows.

\section{Acknowledgement}

The authors gratefully acknowledge the help of Prof. M. K. Aouf in the preperation of this paper and also the referee for his valuable suggestions. This work is supported by UGC grant No.F 24-13/92 (CD-2).

\section{References}

[1] L. V. Ahlfors, Complex Analysis, McGraw Hill Book Co, Inc., New York, 1953.

[2] Z. Nehari, Conformal Mapping, Dover Publications, Inc., New York, 1975.

[3] Susheel Chandra, "Starlikeness of a bounded analytic function," Bull. Cal. Math. Soc., 81(1989), $42-45$. 